University of Wollongong

Research Online

Faculty of Engineering and Information

Faculty of Engineering and Information

Sciences - Papers: Part A

Sciences

$1-1-2013$

\title{
Parameter identification study of a trilayer conjugated polymer actuator curvature model
}

Emmanuel D. Blanchard

University of Wollongong, eblancha@uow.edu.au

Patrick S. Chen

University of Wollongong

Chuc H. Nguyen

University of Wollongong, chuc@uow.edu.au

Follow this and additional works at: https://ro.uow.edu.au/eispapers

Part of the Engineering Commons, and the Science and Technology Studies Commons

Research Online is the open access institutional repository for the University of Wollongong. For further information contact the UOW Library: research-pubs@uow.edu.au 


\title{
Parameter identification study of a trilayer conjugated polymer actuator curvature model
}

\begin{abstract}
This article investigates the effect of two uncertain parameters on a recent new model of conjugated polymer actuators. These uncertain parameters are the diffusion coefficient (D) and the double-layer capacitance $(\mathrm{C} \mathrm{dl})$, which are difficult to measure directly. The model sensitivity to these parameters is analysed and a parameter estimation study is performed using artificially generated data. The parameter estimation method used in this article is based on a Bayesian cost function, and gives us an insight on how much the estimation can be trusted, which is useful information for the design of controllers. Results indicate that for controllers to be designed effectively using this model, the double-layer capacitance is the best known parameter and should therefore be designed for with greater confidence in its value, while the controller should be much more robust with respect to the diffusion coefficient, which should be treated as a stochastic variable for a certain range of possible values.
\end{abstract}

\section{Keywords}

identification, study, trilayer, conjugated, polymer, actuator, curvature, model, parameter

\section{Disciplines}

Engineering | Science and Technology Studies

\section{Publication Details}

Blanchard, E. D., Chen, P. S. \& Nguyen, C. H. (2013). Parameter identification study of a trilayer conjugated polymer actuator curvature model. IEEE/ASME International Conference on Advanced Intelligent Mechatronics (AIM) (pp. 1108-1113). IEEE Xplore: IEEE. 


\title{
Parameter Identification Study of a Trilayer Conjugated Polymer Actuator Curvature Model
}

\author{
Emmanuel D. Blanchard, Patrick S. Chen, and Chuc H. Nguyen
}

\begin{abstract}
This article investigates the effect of two uncertain parameters on a recent new model of conjugated polymer actuators. These uncertain parameters are the diffusion coefficient $(D)$ and the double-layer capacitance $\left(C_{d l}\right)$, which are difficult to measure directly. The model sensitivity to these parameters is analysed and a parameter estimation study is performed using artificially generated data. The parameter estimation method used in this article is based on a Bayesian cost function, and gives us an insight on how much the estimation can be trusted, which is useful information for the design of controllers. Results indicate that for controllers to be designed effectively using this model, the double-layer capacitance is the best known parameter and should therefore be designed for with greater confidence in its value, while the controller should be much more robust with respect to the diffusion coefficient, which should be treated as a stochastic variable for a certain range of possible values.
\end{abstract}

\section{INTRODUCTION}

Recent research into polymeric materials has led to the requirement of reliable prediction models and robust control of Electro Active Polymers (EAP's) as actuators. The credit for the discovery of EAP's is given to Roentgen, who in 1880 , experimented with an electro-activating rubber-band to move a cantilever with a mass attached to the free-end [1]. Since the 1970s academic and industrial interest in EAP applications has sparked research and has increased the list of EAP materials available. The bulk of work focused on the prediction and control models were developed post 1990 [13].

Applications of EAP's are contained in many different areas. Some applications include use as part of electrochromic "smart" glass, as one component in the photoreceptors of electrophotographic and xerographic devices and as thin flexible shaped batteries [4, 5]. Of particular interest is their potential use as an actuator or sensor in a biomimetic situation, commonly referred to as an artificial muscle. One particular group of EAP's known as Conjugated electro active Polymers (CPs) have been attracting the attention of researchers in the past decade. This is mainly due to the features that make them attractive for application including low power consumption, light weight, simple construction and noiseless operation [6]. In particular CPs based on pyrrole, thiophene and aniline are the focus of

E.D. Blanchard is with the school of Mechanical, Materials \& Mechatronic Engineering at the University of Wollongong, NSW 2522, Australia (phone: (+61) 24221 8027; fax: (+61) 24221 3143; e-mail: eblancha@uow.edu.au).

P.S. Chen is with the Faculty of Engineering at the University of Wollongong, NSW 2522, Australia (patrick.s.chen@hotmail.com).

C. H. Nguyen is with the Intelligent Polymer Institute at the University of Wollongong, NSW 2522, Australia (chuc@uow.edu.au). current research. Polypyrrole (PPy) and polyaniline are two of the most commonly used CPs for actuation [7-8].

In order to utilize these EAPs in any application it is highly desirable to have predictive models available for feasibility studies, design optimisation, and precision control. Until recently the control and control-oriented modelling of CPs had been largely unexplored [9]. Original work by J.D.W. Madden [10] and later extended by Fang et al. [11] has led to a model for robust control of CP actuators. These both present a transfer function mathematical model for predicting the bending behaviour of EAPs. The latter work goes on to describe a self-tuning regulator which utilises a parameter projection (in the time domain) step for robust control; this is required because of the relatively short time frame in which the parameters stay constant. Without this parameter estimation step the prediction model becomes inaccurate and results in imprecise control due to the parameters' value expiring. This remedies the previously reported problem of non-repeatability of experiments.

Smith [12] investigated the effect of uncertain parameters for the model used in [11]. He showed that significant discrepancies between the model and its reduced form used for control purposes seemed to indicate that a better suited model would be needed to start developing stochastic controllers. Smith [12] was able to retrieve results with noise using artificial data [12] for the full and reduced models used in [11], but realized that these two models were not able to capture the high frequency phase behaviour, which led to discrepancies in the estimation of uncertainties. His conclusion was that it seemed probable that the biggest problem with the model used in [11] was the discrepancy between experimental data and the model at high frequencies.

In this paper, the techniques used in [12] are applied to a model recently developed by Nguyen et al. [13] which is a variation of the model presented in [11] that proved to better match experimental data at high frequencies. Let's note that this model uses the curvature of the actuator as an output rather than its displacement. However, there is a simple relationship between the two, and this model can therefore easily be compared with the model used in [11]. Parameter estimation is performed using artificially generated data, which yielded similar results between the full model and its reduced form, hinting at an efficient model for control purposes. This work will need to be validated using experimental data. 


\section{Electro-Chemo-Mechanical Modelling of A TRILAYER CP ACTUATOR}

\section{A. The Infinite-Dimensional Model}

The work of Nguyen et al. [13] extends the work of Fang et al. [11] which extended the diffusive-elastic-metal model of Madden [10] for a trilayer conjugated polymer actuator. The model used in [13] combines both the electrochemical and the mechanical dynamics and is thus known as an electro-chemo-mechanical model. The model for the displacement of the actuator is presented in three modules; electrical admittance, electromechanical coupling and mechanical output. The admittance module relates the input voltage to a current flowing through the system. The electromechanical coupling then relates the current in the system to an electrically induced strain and charge density. The final mechanical output module relates the electrically induced curvature to the geometric curvature, thus giving the displacement, as shown in Fig. 1. The equivalent circuit of the trilayer conducting polymer actuator is shown in Fig. 2.

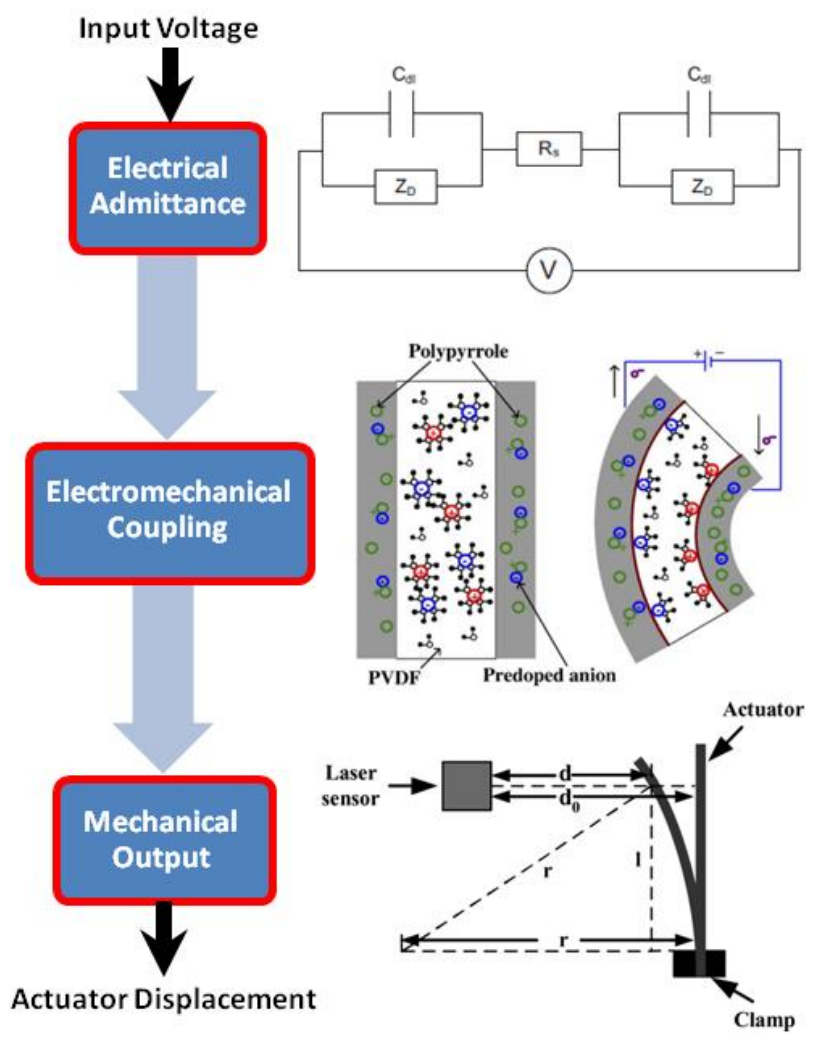

Fig.1. Three distinct modules that form the actuator displacement mathematical model (figure adapted from various figures in [11] and [13])

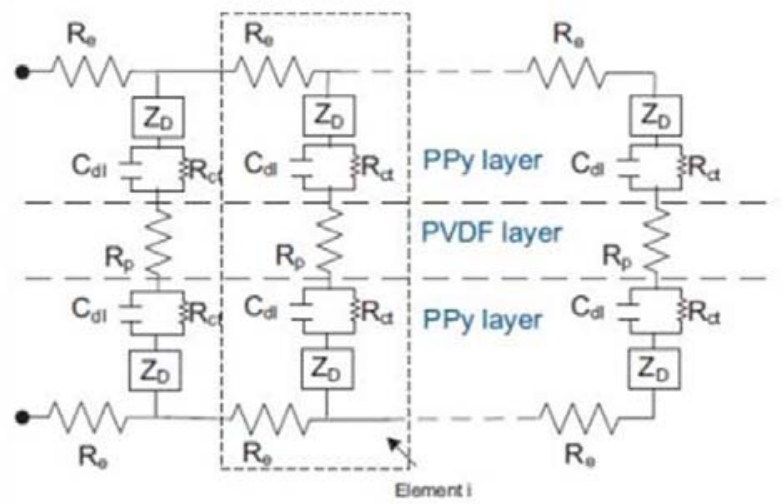

The trilayer actuator is assumed to consist of $n$-elem ents of impedance along its length

Fig.2. Transmission line circuit of the trilayer conducting polymer actuator (adapted from [13])

The transfer function between the input voltage and the curvature of the actuator for that model is given by,

$$
G(s)=\frac{\kappa(s)}{V(s)}=K_{c} \frac{\tanh \left(L \sqrt{2 R_{e} / Z_{i}(s)}\right)}{s \sqrt{2 R_{e} / Z_{i}(s)}}
$$

where

$$
\begin{gathered}
K_{c}=\frac{3 \alpha}{2 h_{p v d f} h W L} \frac{\left(1+\left(h / h_{p v d f}\right)\right)^{2}-1}{\left(1+\left(h / h_{p v d f}\right)\right)^{3}+\left(E_{p v d f} / E_{p p y}\right)-1} \\
Z_{i}(s)=R_{p}+2\left(Z_{d}+\frac{R_{c t}}{1+s R_{c t} C_{d l}}\right)
\end{gathered}
$$

and where (more details can be found in [13])

$\kappa(s)$ is the Laplace transformed curvature of the actuator

$V(s)$ is the Laplace transformed voltage function

$C_{d l}$ is the double-layer capacitance

$D$ is the diffusion coefficient for modelling the diffusion of ion concentration

$R_{e}$ is one element of PPy layer

$R_{p}$ is the resistance of one element of polyvinylidene fluoride (PVDF) layer

$R_{c t}$ is the charge transfer resistance

$L$ is the length of the PPy layer

$W$ is the width of the PPy layer

$h$ is the thickness of the polymer (PPy) layer

$h_{p v d f}$ is the thickness of the PVDF layer

$E_{p p y}$ is the modulus of elasticity for the PPy layer

$E_{p v d f}$ is the modulus of elasticity for the PVDF layer 


\section{B. Reduced Finite-Dimensional Model}

The work of Nguyen et al. [13] also presents a reduced form of the model. Due to the hyperbolic tangent term, the infinite-dimensional system is not suitable for real-time control purposes. The hyperbolic tangent term is therefore approximated by using the equality

$$
\frac{\tanh \left(\frac{1}{2} \sqrt{\frac{X}{Y}}\right)}{4 \sqrt{X Y}}=\sum_{n=0}^{\infty} \frac{1}{X+\pi^{2}(2 n+1)^{2} Y}
$$

and letting $X=1 / Z_{i}$ and $Y=1 /\left(8 R_{e} L^{2}\right)$, which yields a transfer function of the form [13]

$$
G(s) \approx \frac{\sum_{i=0}^{N-1} b_{i} s^{i}}{\sum_{i=0}^{N-1} a_{i} s^{i}}
$$

where the coefficients $b_{i}$ 's and $a_{i}$,'s can be written in terms of the physical constants [13].

For typical parameter values such as the values shown in Table 1, Nguyen et al. showed that $N=9$ is enough to yield very similar transfer functions than the ones observed experimentally. Note that the relationships between $R_{e}$ and $R_{p}$ and to the conductivities $\sigma_{e}$ and $\sigma_{p}$ are given by

$$
R_{e}=\frac{1}{\sigma_{e}} \frac{1}{W \cdot h}, \quad R_{p}=\frac{1}{\sigma_{p}} \frac{2 h_{p}}{W},
$$

TABLE I. Typical PARAmeter VAlues of THE Model

\begin{tabular}{|l|l|l|l|}
\hline Parameter & \multicolumn{1}{|c|}{ Value } & Parameter & \multicolumn{1}{c|}{ Value } \\
\hline$D$ & $5.1344 \times 10^{-13} \mathrm{~m}^{2} \cdot \mathrm{s}^{-1}$ & $W$ & $2.6 \times 10^{-3} \mathrm{~m}$ \\
\hline$C_{d l}$ & $5.1189 \mathrm{~F}^{-1}$ & $h$ & $30 \times 10^{-6} \mathrm{~m}$ \\
\hline$\sigma_{e}$ & $3 \times 10^{3}{\mathrm{~S} . \mathrm{m}^{-1}}$ & $h_{p}$ & $55 \times 10^{-6} \mathrm{~m}$ \\
\hline$\sigma_{p}$ & $8{\mathrm{~S} . \mathrm{m}^{-1}}$ & $h_{p v d f}$ & $110 \times 10^{-6} \mathrm{~m}$ \\
\hline$R_{c t}$ & $10.6 \times 10^{3} \Omega$ & $E_{p p y}$ & $200 \mathrm{MPa}$ \\
\hline$L$ & $14 \times 10^{-3} \mathrm{~m}$ & $E_{p v f}$ & $117 \mathrm{MPa}$ \\
\hline
\end{tabular}
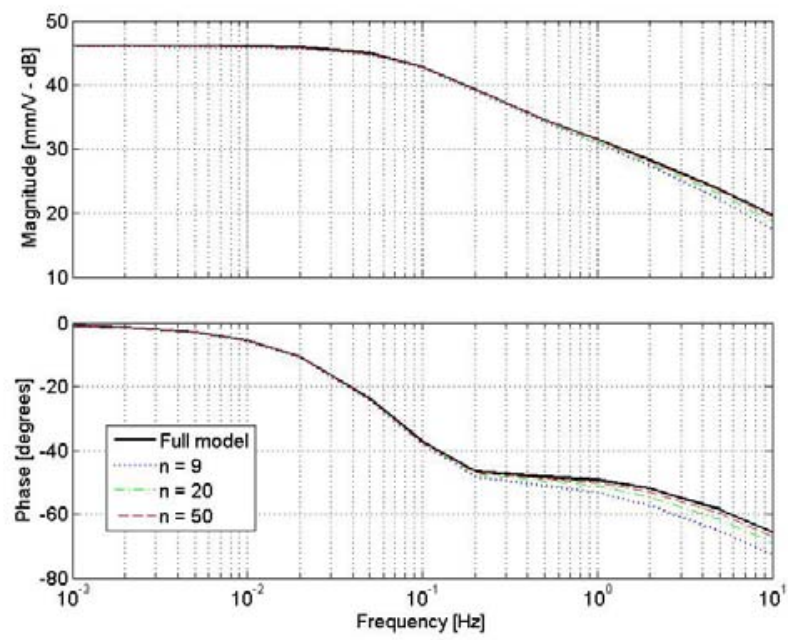

Figure 3: Sensitivity of Bode plots to the number of coefficients used in the model
Fig. 3 shows the sensitivity of Bode plots to the number of coefficients used in the model. Fig. 4 shows the sensitivity of Nyquist diagram to the model, which is relevant since the Bayesian cost function used later this study will be based on the graphical representation complex numbers associated to the transfer function.

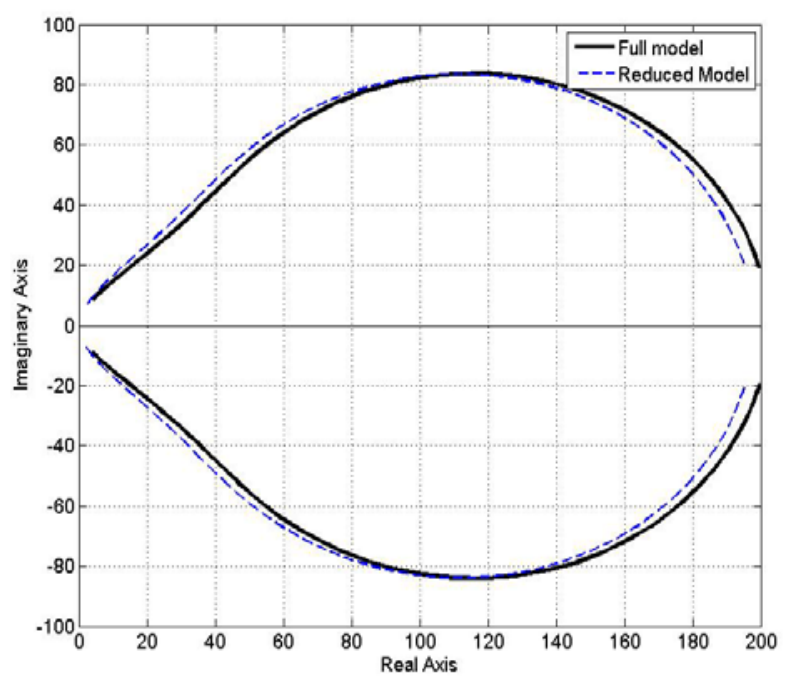

Figure 4: Sensitivity of the Nyquist diagram to the model

\section{Sensitivity OF THE Model to PARAMEter VARIATION}

Two parameters are considered uncertain. The diffusion coefficient $(D)$ and the double-layer capacitance $\left(C_{d l}\right)$ are uncertain due to the fact that they depend on external conditions. $D$ tends to vary more due to the fact that it decreases due to solvent evaporation which hinders the diffusion of ions. This is the main reason for the very large variability of $D$. The expected ranges of these three uncertain parameters are given in Table 2.

TABLE II. PhysicAlly MeANingful VAlues For the UNCERTAiN PARAMETERS

\begin{tabular}{|l|l|l|}
\hline Parameter & \multicolumn{1}{|c|}{ Minimum Value } & \multicolumn{1}{c|}{ Maximum Value } \\
\hline$D$ & $10^{-14} \mathrm{~m}^{2} \mathrm{~s}^{-1}$ & $10^{-10} \mathrm{~m}^{2} \mathrm{~s}^{-1}$ \\
\hline$C_{d l}$ & $3 \mathrm{~F} \mathrm{~m}^{-1}$ & $7 \mathrm{~F} \mathrm{~m}^{-1}$ \\
\hline
\end{tabular}

A sensitivity analysis is performed by plotting Bode plots for different values of the uncertain parameters for both models. The sensitivity of Bode plots to variation in the double-layer capacitance $C_{d l}$ is shown in Fig. 5. The sensitivity of Bode plots to variation in the diffusion coefficient variation $D$ is shown in Fig. 6. These results indicated that $C_{d l}$ caused both of the models to vary the most. 
The sensitivity analysis results and implications are used as a means of "checking" the estimated parameter values, which is achieved by assessing the consistency of the parameter estimation results with the results observed when plotting the Bode plots for different values of the uncertain parameters.
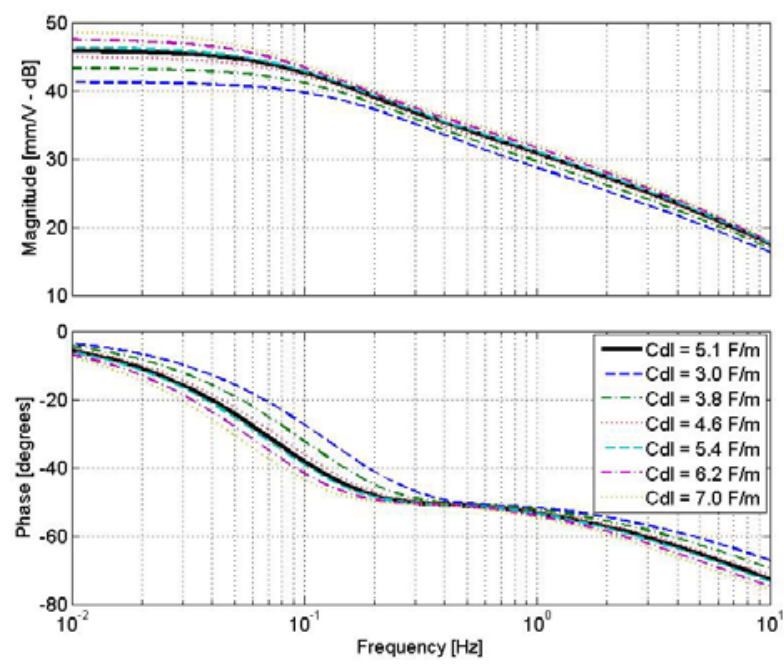

Figure 5: Sensitivity of Bode plot to variation in the double-layer capacitance $C_{d l}$ using the reduced model $(\mathrm{N}=9)$

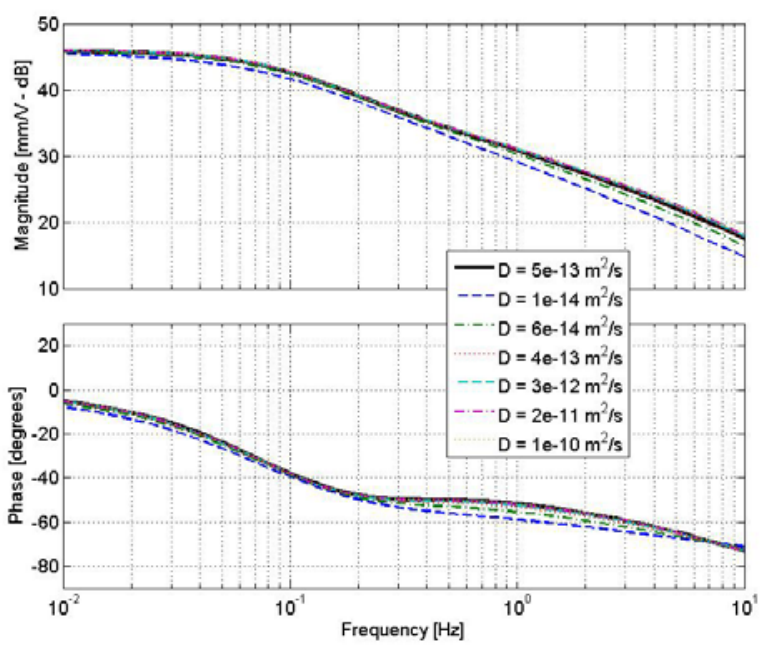

Figure 6: Sensitivity of Bode plot to variation in the diffusion coefficient $D$ using the reduced model $(\mathrm{N}=9)$

\section{PARAmeter Estimation Using FreQUenCy RESPONSE DATA}

\section{A. Choice of Parameter Estimation Method}

A Bayesian approach to parameter estimation is used. The simulation involves the formation of a multidimensional grid, calculating the value of the cost function at every point considered, and finding the minimum of those calculated values. The main advantage of this parameter estimation is that the quality of the maximum likelihood estimate is related to the shape of the Bayesian cost function, with a sharp minimum indicating an accurate estimate [13]. This will be useful in order to design better controllers for artificial muscles. Adaptive controllers are typically designed based on estimates for the uncertain parameter without information on how reliable these estimates are. This is especially a problem when dealing with non-identifiability issues, i.e. when several combinations of values for the uncertain parameters basically yield the same responses. In these cases, stochastic controllers should be designed instead, using a range of possible values for these uncertain parameters and trying to obtain the best average answer since the actual values of these parameters cannot be known. Some parameters might still have estimates which can be trusted while others cannot. This can be visualized when looking at the shape of the Bayesian cost function. The cost function can yield very similar values when a first parameter varies (this parameter is unidentifiable) while it yields very different values when another (identifiable) parameter varies, as explained in [13]. In that case, the stochastic controller would only need to take the parameters that cannot be estimated into account.

For greater precision, MATLAB's constrained minimisation function ("fmincon") was used. This involves providing an initial estimate and bounds to constrain the problem. The results from the grid simulation are used as the initial estimate, and bounds proportional to these results were used as the bounds. This parameter estimation method had already been tested successfully with a number of sets of artificially generated data obtained by introducing white noise into a previous model [12].

\section{B. Choice of Cost Function}

A traditional choice of cost function is the sum of square residuals in the ordinates only (7), that is the sum of the square of the differences between the observed and predicted " $y$-values". This choice is unsuited to this particular inverse problem because a method of combining the cost function for both the magnitude and phase results is needed. This disregards that the two plots are linked as the two plots are a graphical representation of one complex number.

$$
\varepsilon=\sum_{n}^{d e f} w_{M}\left|M\left(j \omega_{n} ; R ; D ; \delta\right)-M_{n}\right|^{2}+w_{\phi}\left|\phi\left(j \omega_{n} ; R ; D ; \delta\right)-\phi_{n}\right|^{2}
$$

where

$\omega_{n}$ is the $n$-th discrete frequency at which the magnitude and phase measurements were taken

$M\left(j \omega_{n} ; R ; D ; \delta\right)$ is the simulated magnitude data using particular parameter values for $R, D$ and $\delta$. Either of the full or reduced model can be used here. 


\section{$M_{n}$ is the $n$-th magnitude measurement}

$w_{M}$ is the weight for the magnitude residuals. This needs to be chosen so that its units are the reciprocal of the units of $M$ and so that equal priority is given to both $M$ and $\phi$

$\phi\left(j \omega_{n} ; R ; D ; \delta\right)$ is the simulated magnitude data using particular parameter values for $R ; D$ and $\delta$. Either of the full or reduced model can be used here.

\section{$\phi_{n}$ is the $n$-th phase measurement}

$w_{\phi}$ is the weight for the phase residuals. This needs to be chosen so that its units are the reciprocal of the units of $\phi$ and so that equal priority is given to both $M$ and $\phi$.

For this reason alone it is far more mathematically rigorous to choose a cost function that represents the difference between the observed complex number and the predicted complex number. In effect this would mean that the process would be "curve-fitting" the Nyquist plot of the measured experimental data with either or both of the full and reduced models. That is, the cost function is defined as

$$
\varepsilon=\sum_{n}\left|G\left(j \omega_{n} ; R ; D ; \delta\right)-M_{n} \exp \left(j \phi_{n}\right)\right|^{2}
$$

where $G\left(j \omega_{n} ; R ; D ; \delta\right)$ is the simulated data using particular parameter values for $R, D$ and $\delta$. Either of the full model or reduced model can be used here.

\section{Results}

Artificial data were created based on the full model, and by adding artificially generated noise of $2.5 \%$ on the values of magnitudes and phases to simulate noisy measurements. The sample frequencies used as "measurements" in order to generate the Bayesian cost functions were the following: $0.001 \mathrm{~Hz}, 0.002 \mathrm{~Hz}, 0.005 \mathrm{~Hz}, 0.01 \mathrm{~Hz}, 0.02 \mathrm{~Hz}, 0.05 \mathrm{~Hz}$, $0.1 \mathrm{~Hz}, 0.2 \mathrm{~Hz}, 0.5 \mathrm{~Hz}, 1 \mathrm{~Hz}, 2 \mathrm{~Hz}, 5 \mathrm{~Hz}$, and $10 \mathrm{~Hz}$.

Results for both the reduced model and the full model are displayed in Table 3, which shows that in the absence of noise, the full model retrieves the exact values of $D$ and $C_{d l}$, i.e. $D=5.1344 \times 10^{-13} \mathrm{~m}^{2} . \mathrm{s}^{-1}$ and $C_{d l}=5.1189 \mathrm{~F}^{-\mathrm{m}^{-1}}$, while the reduced model has a significant error for the estimation of $D$. With artificially generated noise of $2.5 \%$ on the values of magnitudes and phases, the difference between the estimates for each of the two models is not very significant. It indicates that the reduced model is suitable, as it is not different from the full model, and due to the fact that errors for the estimation of $D$ are expected given the shape of the cost functions. This can be explained by looking at Fig. 7, which shows the cost function plot results using the full model and Fig. 8, which shows the cost function plot results using the reduced model. The figures reveal that non-identifiability issues exist for both the full and reduced models as evidenced by the long troughs that appear in the plots of the cost function (as explained earlier in this paper, and detailed in [13]). Both figures clearly show that $D$ is non-identifiable, and should therefore be treated as a stochastic parameter for control purposes. A control algorithm should only attempt to estimate $C_{d l}$.

The full model is not suitable for real-time control purposes, but the reduced model is suitable. Significant discrepancies between the two models would have shown that the estimates of the uncertain parameters might not necessarily result in good controllers even if the Bayesian cost functions have sharp minima indicating accurate estimates. Controller gains would be calculated based on these estimates, but the fact that the reduced model would yield different estimates would mean that the controller (which is based on a reduced model) would have an effect on the system somewhat different than what we might expect. Such a case occurred with models previously studied and their reduced versions [12]. With this latest model, the reduced model seems to be very suitable for control applications. Therefore, this parameter estimation study shows us that using the model developed by Nguyen et al. [13] might improve the control performance obtained in [11].

TABLE III. ESTIMATES FOR THE UNCERTAIN PARAMETERS FOR THE LABORATORY RECORDED DATA

\begin{tabular}{|c|c|c|}
\hline Model & Estimate for $C_{d l}$ & Estimate for $D$ \\
\hline $\begin{array}{l}\text { Full model - } \\
\text { no noise }\end{array}$ & $\begin{array}{l}\text { 5.1189 F.m }{ }^{-1} \\
\text { (error: } 0 \% \text { ) }\end{array}$ & $\begin{array}{l}5.1344 \times 10^{-13} \mathrm{~m}^{2} \cdot \mathrm{s}^{-1} \\
\text { (error: } 0 \% \text { ) }\end{array}$ \\
\hline $\begin{array}{l}\text { Reduced model - } \\
\text { no noise }\end{array}$ & $\begin{array}{l}5.2238 \text { F. } \mathrm{m}^{-1} \\
\text { (error: } 2.0 \% \text { ) }\end{array}$ & $\begin{array}{l}5.7693 \times 10^{-13} \mathrm{~m}^{2} \cdot \mathrm{s}^{-1} \\
\text { (error: } 12.3 \%)\end{array}$ \\
\hline $\begin{array}{l}\text { Full model - } \\
\text { with } 2.5 \% \text { noise }\end{array}$ & $\begin{array}{l}\text { 5.6257 F.m } \\
\text { (error: } 9.9 \% \text { ) }\end{array}$ & $\begin{array}{l}8.3023 \times 10^{-13} \mathrm{~m}^{2} \cdot \mathrm{s}^{-1} \\
\text { (error: } 61.7 \%)\end{array}$ \\
\hline $\begin{array}{l}\text { Reduced model - } \\
\text { with } 2.5 \% \text { noise }\end{array}$ & $\begin{array}{l}5.7229 \text { F.m } \\
\text { (error: } 11.8 \% \text { ) }\end{array}$ & $\begin{array}{l}8.5180 \times 10^{-13} \mathrm{~m}^{2} \cdot \mathrm{s}^{-1} \\
\text { (error: } 65.9 \%)\end{array}$ \\
\hline
\end{tabular}

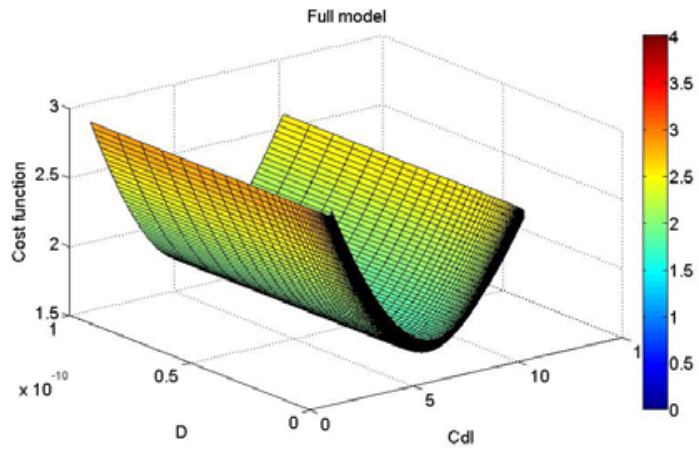

Figure 7: Cost function plot results using the full model

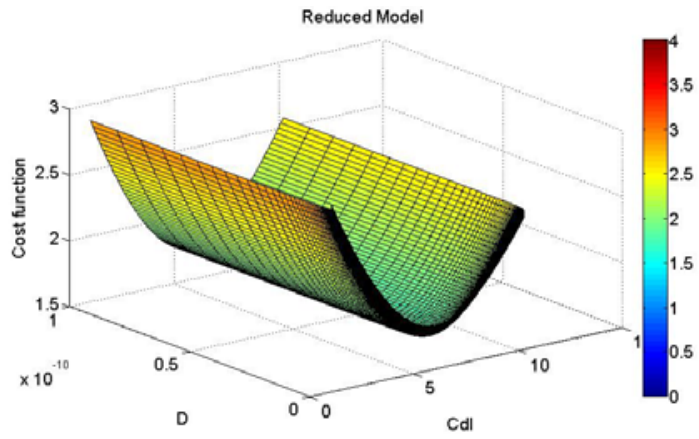

Figure 8: Cost function plot results using the reduced model 
From the sensitivity analysis it was concluded that $C_{d l}$ affects the behaviour of both of the models more than $D$. In contrast the diffusion coefficient $D$ shows signs of nonidentifiability, which is coherent with the implications from the sensitivity analysis that $C_{d l}$ is the most influential parameter on the shape of the Bode plots.

The implications from the results obtained in the analysis of the parameter estimation results are that in a conservative design of controllers the value of $C_{d l}$ is the only parameter that can be designed for confidently. The results also show that $D$ would need further study to estimate confidence intervals around the estimated value so that designs can be adequately conservative. A study in to achieving confidence intervals for the parameters estimated would require more sophisticated parameter estimation techniques.

\section{CONCLUSIONS}

The objective of this study was to find non-identifiability issues using the tri-layered conjugated polymer actuator displacement model used in [13] in order to treat estimates that can be trusted as deterministic and use stochastic formulations when dealing with estimates that cannot be trusted (when the Bayesian cost function has an entire region of minima, e.g., a line or a valley). The two uncertain parameters are the diffusion coefficient $(D)$, and the doublelayer capacitance $\left(C_{d l}\right)$, which are difficult to measure directly. The parameter estimation method used in this study was a Bayesian approach similar to the one developed by Blanchard et al. $[14,15]$ which has been proven to identify zones of non-identifiability [14]. Since the full model used in [13] is not suitable for real-time control purposes and is approximated by a reduced form of the model [13]. The similar results obtained with both models indicates that the model developed by Nguyen et a. [13] might be an efficient model for control purposes with the potential of improving results obtained in [11].

Results indicate that for controllers to be designed effectively using this model, the double-layer capacitance is the best known parameter and should therefore be designed for with greater confidence in its value, while the controller should be much more robust with respect to the diffusion coefficient, which should be treated as a stochastic variable for a certain range of possible values.

The results presented in his paper will need to be validated using experimental data. Future work will also include the use of the polynomial chaos theory coupled with the Bayesian approach. Typical runtimes for the resolutions shown in Figs. 7-8 (grids of $200 \times 200 \times 200$ ) were between 15 and 30 minutes (13 sample frequencies were used). With the polynomial chaos theory, results for a similar resolution would probably easily be obtained within a few seconds or even less $[14,15]$, which would also enable the use of higher sample frequencies if needed.

\section{REFERENCES}

[1] Bar-Cohen, Y., Electroactive Polymers (EAP) as Artificial Muscles, von Karman Auditorium Lecture Series, , 2002, accessed 22/3/2012, http://trs-new.jpl.nasa.gov/dspace/bitstream/2014/11817/1/02-472.pdf

[2] Bar-Cohen, Y. (ed.), Electroactive polymer (EAP) actuators as artificial muscles: reality, potential, and challenges, 2nd ed., SPIE The International Society for Optical Engineering, Washington, USA, 2004.

[3] National Research Council (U.S.) Committee on Polymer Science and Engineering, "Advanced Technology Applications", in Polymer Science and Engineering: the shifting research frontiers, National Academy Press, Washington, D.C., USA, 1994.

[4] Blythe, A.R. \& Bloor, D., "Applications of electro-active and conductive polymers", in Electrical Properties of Polymers, 2nd ed. Cambridge University Press, New York, USA, 2005.

[5] Scrosati, B (ed.), Applications of Electroactive Polymers, Chapman \& Hall, London, 1993.

[6] Alici, G., "An effective modelling approach to estimate nonlinear bending behaviour of cantilever type conducting polymer actuators", Sensors and Actuators B: Chemical, vol. 141, no. 1, pp. 284-292, 2009.

[7] Madden, PGA., Madden, JDW., Anquetil, PA., Vandesteeg, NA., \& Hunter, IW., "The Relation of Conducting Polymer Actuator Material Properties to Performance", IEEE Journal of Oceanic Engineering, vol. 29, no. 3, pp. 696-705, 2004.

[8] Smela, E., "Conjugated Polymer Actuators for Biomedical Applications", Advanced Materials, vol. 15, no. 6, pp. 481-494, 2003

[9] Fang, Y., Tan, X., Shen, Y., Xi, N., \& Alici, G., “A scalable model for trilayer conjugated polymer actuators and its experimental validation", Materials Science and Engineering C: Materials for Biological Applications, vol. 28, no. 3, pp. 421-428, 2008.

[10] Madden, J.D.W., "Conducting Polymer Actuators', PhD thesis, Department of Mechanical Engineering, Massachusetts Institute of Technology, 2000

[11] Fang, Y., Tan, X., \& Alici, G., "Robust Adaptive Control of Conjugated Polymer Actuators", IEEE Transactions on Control Systems Technology, vol. 16, no. 4, pp. 600-612, 2008.

[12] Smith, M.J., "A Parameter Identification Study of Frequency Response Data for a Tri-Layered Conjugated Polymer Actuator Displacement Model', Undergraduate Thesis, University of Wollongong, November 2012.

[13] Nguyen, C.H., Alici, G., and Wallace, G.G., "Modelling trilayer conjugated polymer actuators for their sensorless position control", Sensors and Actuators A-Physical, ISSN 0924-4247, 10/2012, vol. 185 , pp. $82-91,2012$

[14] Blanchard, E. D., Sandu, A. \& Sandu, C., "Parameter estimation for mechanical systems via an explicit representation of uncertainty", Engineering Computations: international journal for computer-aided engineering and software, vol. 26, no. 5, pp. 541-569, 2009.

[15] Blanchard, E. D., Sandu, A. \& Sandu, C., "Polynomial chaos-based parameter estimation methods applied to a vehicle system". Proceedings of the Institution of Mechanical Engineers, Part K: Journal of Multi-body Dynamics, vol. 224, No. 1, pp. 59-81, 2010. 